\title{
Year 2020: How Will It Impact Identities of Children and Youth over Time?
}

\author{
Nazilla Khanlou ${ }^{1}$ (D)
}

Accepted: 1 January 2021 /Published online: 20 January 2021

(C) The Author(s), under exclusive licence to Springer Science+Business Media, LLC part of Springer Nature 2021

\begin{abstract}
In this Editorial, as the Guest Editor to the Special Issue on Youth Identity, I reflect on the year 2020. The year was a challenging one in relation to the global impacts of the COVID-19 pandemic, as well as the continued inequities and systemic injustices faced by racialized populations. As an intersectionality-informed identity researcher, I argue that because of the complexity of our individual and collective identities, identity scholarship is not limited to a particular discipline, or theory, or methodology.
\end{abstract}

Keywords Identity $\cdot$ COVID-19 pandemic $\cdot$ Social movements $\cdot$ Intersectionality $\cdot$ Youth Editorial

As the epidemic year of 2020 draws to end, I find myself reminiscing the closing days of 1999. For those of us who remember it, that was a year which was predicted to lead to a global computer problem: the Y2K bug. The Y2K bug referred to potential computer malfunction because of how calendar dates were formatted and stored (in six digits instead of eight) (Aspinwall et al. 2005; McKinney and Mulvin 2019). As the impending 2000 calendar year approached closer, there was widespread speculation of Y2K's impact on all related technology. Y2K was thought to affect our daily technologically driven lives dramatically and suddenly as the clock ushered in the year 2000 . In preparation for the possible breakdown of technology, advise was given from different sectors on how to prevent or prepare for it. For example, in Canada as it was the winter season, a survival kit of sorts was recommended by some. I confess I prepared one, much to the amusement of my experienced engineer father, but with the support of my more recently graduated engineer brother, and the encouragement of my concerned educator mother. Items like candles, matches, batteries, water, some canned foods, and such were part of the kit.

As I celebrated the New Year's Eve of that year with family, we wondered what the next day might hold. The next day came. Nothing significant seemed to have occurred. My desktop

Nazilla Khanlou

nkhanlou@yorku.ca; https://nkhanlou.info.yorku.ca/

1 Women's Health Research Chair in Mental Health, Faculty of Health, School of Nursing, York University, 4700 Keele Street, Toronto, ON, Canada 
computer still worked, and no files were lost. That was a major relief. There were no smart phones to worry about back then nor the many digitally interconnected technologies that we are becoming increasingly dependent on in our homes and workplaces.

Twenty years later, the reverse seems to have occurred. No one (that I personally know of) could predict in late December of 2019 the type of year 2020 would turn out to unfold. The COVID-19 pandemic is still here, both locally and globally. The pandemic is now well into its second wave. Lives are continuing to be lost as pandemic directives are communicated and regulated. Much has been written on the COVID-19 pandemic in the span of 1 year. Researchers from different disciplines are rapidly contributing to a growing body of health and social sciences literature on it, including recent publications in the International Journal of Mental Health and Addiction. As a mental health researcher, during the earlier days of the pandemic in North America, I wrote about the need for a public mental health system in Canada (Khanlou 2020). Despite the increasing mental health toll, we do not yet have a public mental health system. Soon, it became evident that the pandemic was impacting marginalized populations disproportionately. The underlying inequities in the social determinants of health were exacerbated by the pandemic and, in turn, placed marginalized populations at greater risk of pandemic-related health disparities. For example, the pandemic had led to increases in gender-based violence against women, with systemic barriers needing to be addressed to provide upstream mental health support for racialized women (Khanlou et al. 2020).

Inequities and the social disparities of the year 2020 were not confined to the pandemic. Discrimination, systemic injustices, and racism directed at Black, Indigenous, and minority communities led to renewed social movements, demonstrating the agency of youth and young community leaders. The Black Lives Matter movement grew in the USA and quickly became a global movement. Social media reflected the mounting societal response to systemic and sustained violence against Black and Indigenous communities in such hashtags as \#SayTheirNames, \#SayHerName, and \#IndigenousLivesMatter. As the social movements underscored and taught younger (and older) generations, our identities are not neutral or solely psychological or context-free. They are political, some are privileged, and others become a target for violence the moment one steps outside of home.

Understandably and importantly then, to account for the complexity and continuing pertinence of exploring our individual and collective identities, scholarship on identity is not confined to one discipline, theory, or methodology. As the range of articles in this issue of the International Journal of Mental Health and Addiction demonstrate, identity research continues to develop and inform us, as does our collective reflection on its relevance to mental health and wellbeing. For example, Christmas examines normativity in relation to youth identity and resilience through a critical interpretive synthesis. Through their scoping review, Epstein and colleagues consider the experiences of nursing students who identify with a disability and instructors in nursing practice. Using semi-structured interviews, Islam and colleagues examine the perspectives of Bangladeshi immigrant parents on youth identity and how these differ from that of the parents. Quantitative findings of a mixed-method study on immigrant and Canadian-born Pakistani youth's identity, self-esteem, and mental health are presented by Khan and colleague. Drawing from critical and intersectional perspectives, Nunes provides an overview of different approaches to research on youth cultural identity and discusses research opportunities. Identity-related factors impacting African immigrant youth's mental health in Canada are considered by Olawo and colleagues, based on an interpretive description study. Through a descriptive qualitative approach, $S u$ and colleagues examine the stressors, social support, and traditional cultural beliefs of Chinese immigrant mothers of children with 
developmental disabilities in Canada. In another article, $\mathrm{Su}$ and colleague investigate Chinese and Canadian identity responses in relation to experiences of shame and guilt.

As an intersectionality-informed identity researcher, I wonder how the pandemic and social movements of the 2020 will impact the identities of children and youth over time. How will the future adults of tomorrow reflect on this period of their lives? Will their identities have preand post-year 2020 as reference points in relation to their self-concept, their sense of belonging to their communities, and integration to society at large? Will the significant individual and collective challenges of 2020 lead to sustained efforts to address structural inequities and systemic exclusion of "non-mainstream" identities? Time will tell.

\section{Compliance with Ethical Standards}

Conflicts of Interest The author declares that she has no conflict of interest.

Research Involving Human Participants This submission is the Editorial for the International Journal of Mental Health and Addiction's Special Issue on Youth Identity. It does not report on research involving human participants.

Informed Consent Informed consent was not required as this submission is an Editorial as per above.

\section{References}

Aspinwall, L. G., Sechrist, G. B., \& Jones, P. R. (2005). Expect the best and prepare for the worst: anticipatory coping and preparations for Y2K. Motivation and Emotion, 29(4), 353-384.

Khanlou, N. (2020). Editorial: call for a Canadian public mental health system: transformative change amid a global pandemic. Journal of Concurrent Disorders, 2(1), 1-2 Available url: https://concurrentdisorders.ca/ 2020/03/31/call-for-a-canadian-public-mental-health-system/.

Khanlou, N., Ssawe, A., Vazquez, L. M., Pashang, S., Connolly, J. A., Bohr, Y., Epstein, I., Zahraei, S., Ahmad, F., Mgwigwi, T., \& Alamdar, N. (2020). COVID-19 pandemic guidelines for mental health support of racialized women at risk of gender-based violence: Knowledge synthesis report. Funded by Canadian Institutes of Health Research (CIHR) Operating Grant: Knowledge Synthesis: COVID-19 in Mental Health \& Substance Use. York University Available url: https://cihr-irsc.gc.ca/e/52062.html.

McKinney, C., \& Mulvin, D. (December 2019). Bugs: rethinking the history of computing. Communication, Culture and Critique, 12(4), 476-498. https://doi.org/10.1093/ccc/tcz039.

Publisher's Note Springer Nature remains neutral with regard to jurisdictional claims in published maps and institutional affiliations. 